\title{
A HOMOTOPY METHOD FOR STABILIZATION OF MUITTIVARIABLE SYSTEMS WITH STRUCTURAL CONSTRAINTS ON STATE FEEDBACK
}

\author{
Makarand S. Phatak and S. Sathiya Keerthi \\ Department of Computer Science\& Automation \\ Indian Institute of Science \\ Bangalore 560012 INDIA
}

\begin{abstract}
Various structural Constraints on the state feedtack gain matrix, $K$, used in the control of a multivariable dynamical system arise because of the way feedback is realized. Static output feedback, decentralized state feedback, simultaneously stabilizing state feedback for several operating points of a system, and two-controller feedback are some examples of control structure realizations which impose constraints on $\mathrm{K}$. We cast each of the problems in a general class of problems of stabilization of multivariable systems with such structural constraints into a problem of solving a system of equalities and inequalities which is then solved using a continuous realization of the gradient projection method and a barrier method under the homotopy framework. Our method is illustrated with two examples.
\end{abstract}

\section{Introduction}

Various structural constraints on the state feedback gain matrix, $K$, used in the control of a multivariable dynarnical system arise because of the way feedback is realized. For example, consider the case of static output feedback. The output feedback and the state feedback gain matrices are related by a simple linear relationship which specifies the structural constraints on $K$. Decentralized state feedback, simultaneously stabilizing state feedback for several operating points of a system and two-controller feedback are three more examples of control structure realizations which impose constraints on $K$.

It is well known that the eigenvalues of a controllable system can be located anywhere in the complex plane by state feedback. However when there are control structure constraints on the state feedback the freedom of arbitrary eigenvalue assigi:ment is lost. Under control structure constraints on the state feedback there are two problem formulations. The first one is to find when arbitrary eigenvalue assignment is still possible. The iecond one is to find when eigenvalues can be located in some des red region in the complex plane. We consider in this paper the second formulation and give a numerical method which treats a general class of stabilization problems in a single framework, and has good empirical success.

In our method we formulate each of the stabilization problems as a problem of sclving a system of equalities and inequalities. The structural constraints on the state feedlback specify the equalities. For satisfactory dynamical behaviour, the closedloop eigenvalues must be in some desired stability region, $S$, in the complex plane. The inequalities correspond to this requirement. The coefficients of the closed-loop character stic matrix polynomial and the coefficients of quadratic and linear factors of the closed-loop characteristic scalar polynomial form the set of variables. This set is not minimal as the variables in the set are constrained by certain equalities. These equalities are appended to the set of equalities specified by the control structure constraints. To solve the system of equalities and iriequalities we employ a homotopy framework which also uses a ba rier function and a continuous realization of the gradient project ion method.

The stabilization problems mentioned here have also been considered in several earlier papers. We only cits the related ones here. A solution to the output feedback stabilization problem is proposed in $[5,12]$. The problem of simultane sus stabilization of several operating points using state feedback is studied in [1]. The problem of decentralized state feedback stabilization of large-scale interconnected systems is considered in $[i, 11,13]$. Our method is fundamentally different from the method:, in the above papers.

Homotopy methods have been used before for eigenvalue assignment by static outpet feedback and decentralized state feedback $([4,10])$. Their aim as well as their homotopy formulation and solution are very different from ours. Assuming certain sufficient conditions which imply the solvability of the arbitrary eigentvalue assignment problems (and not stabilization problem:.) to hold, they devise special homotopies with guaranteed success.

The paper is organised as fololows. In sectiol 2, we show how members of a class of important constrained stabilization problems can be formulated as problems of solving systems of equalities and inequalities. In Section 3, we describe the methods of gradient projection and barrier functions under the homotopy framework. In Section 4, we present two examples outlining our approach and point out some merits of our method $i$ i comparison with some other methods. We conclude the paper in section 5 .

\section{Problem formulation}

Consider the multivariable system

$$
\dot{x}(t)=A x(t)+B u(t), \quad y(t)=C x(t)
$$

where $x \in R^{\prime \prime}, u \in R^{m}, \mathrm{y} \in R^{p}$, and, $\mathrm{A}, \mathrm{B}, \mathrm{C}$ are constant matrices of appropriate dimensions. We will assume the following throughout : the system (2.1) is controllable and observable, rank $B=m$, and rank $C=p$. The feedback we consider $\mathrm{s}$ of the form

$$
u(t)=-K x(t)
$$

The state feedback gain matrix $K$ is to be chosen such that the closed-loop eigenvalues, i.e., eigenvalues of $(\mathbf{A}-B K)$ are in some 
desired stability region, $S$, in the complex plane. $S$ comes from specifications of the desirable dynamical behaviour is the closedloop system. A closed-loop system with eigenvalues in $S$ will be called as an $S$-stable system. We consider control structure constraints on $K$ and aim to achieve S-stability for the closedloop system. To solve this constrained problem we formulate a system of inequalities and equalities:

$$
\begin{aligned}
& g(v) \leq 0 \\
& h(v)=0
\end{aligned}
$$

where $v$ is some vector of intermediate variables. $C$ ce a $v$ atitisfying (2.3) and (2.4) is found the feedback gain mi rix reqi $x^{1}$ for stabilization can be easily found. In Section 2.1 we show $]$ $v$ is chosen and (2.3) is derived. In Section 2.2, the fualities ( 4) are obtained.

\subsection{Choice of variables and formation of in- equalities}

The feedback gain matrix, $K$, is determined uniquely by the coefficients of the closed-loop characteristic matrix polynomial, $P(s)$ (see [1] and the appendix), where

$$
P(s)=\vec{P}(s)+\Gamma V(s)
$$

where $\bar{P}(s)$ is $m \times m, \Gamma$ is $m \times n$ and $V(s)$ is $n \times m$. We give expressions for $\bar{P}(s)$ and $V(s)$ in the Appendix A. The closedloop scalar characteristic polynomial is given by

$$
\operatorname{det}(s I-A+B K)=\operatorname{det}(P(s)) .
$$

Since $\Gamma$ determines $K$ uniquely its elements can be chosen as variables. However, it is very difficult to generate, inequalities corresponding to the S-stability specification in terns of $\Gamma$. The inequalities can be easily specified in terms of elements of $\mathbf{A}$, the set of closed-loop eigenvalues. However, the eigenvalues are complex, in general. To avoid working with complex numbers one way is to fix the number of closed-loop complex conjugate pairs of eigenvalues and work with the real and imaginary parts which are real. But such a fixation is rigid and will not allow change from a complex conjugate pair of eigenvalues to two real eigenvalues and vice versa. This difficulty can be overcome as follows. Denote the factorization of $\operatorname{det}(s I-\boldsymbol{A}+B K)$ as

$$
\operatorname{det}(s I-A+B K)=\left[\prod_{i=1}^{l}\left(s^{2}+a_{i} s+b_{i}\right) \mid(s-\mathrm{A})\right.
$$

This corresponds to the expansion of $\operatorname{det}(s I-A+B K)$ into $l$ quadratic factors, where $l=\lfloor n / 2\rfloor$. The last term $(s-\mathrm{A})$ occurs only when $n$ is odd. We collect $\gamma_{i, j}$, the elements of $\Gamma$, and the $a_{i}, b_{i}$ and $\lambda$ in $v$, the vector of variables, as

$$
v=\left[\gamma_{1,1}, \ldots, \gamma_{1, n}, \ldots, \gamma_{m, 1}, \ldots, \gamma_{m, n}, a_{1}, b_{1}, a_{2}, b_{2}, \ldots, \lambda\right]^{T}
$$

It is clear that such a set $v$ is not minimal, i. .., it contains more elements than needed to determine $K$ uniquely. The elements of $v$ are constrained by equalities. These constraints come from (2.5)-(2.7) and we shall give expressions for them in section 2.2 .

We now give a set of inequalities for a popular stability region $S$ shown in Fig. 1. The elements of $\Gamma$ do not appear in the inequalities. The set of inequalities which the $\left(a_{i}, b_{i}\right)^{\prime} s$, and the $\lambda$ must satisfy for the closed-loop eigenvalues to lie in $S$ are :

$$
\begin{gathered}
\left.\alpha_{2} \leq-\left(a_{i} / 2\right) \leq \alpha_{1}, \quad b_{i} \leq\left(1+\tan ^{2} \theta\right)\left(a_{i \prime}\right)^{2}\right)^{2} \\
0 \leq \alpha_{1}^{2}+\alpha_{1} a_{i}+b_{i}, \quad 0 \leq \alpha_{2}^{2}+\alpha_{2} a_{i}+l_{i}, \\
\alpha_{2} \leq \lambda \leq \alpha_{1}
\end{gathered}
$$

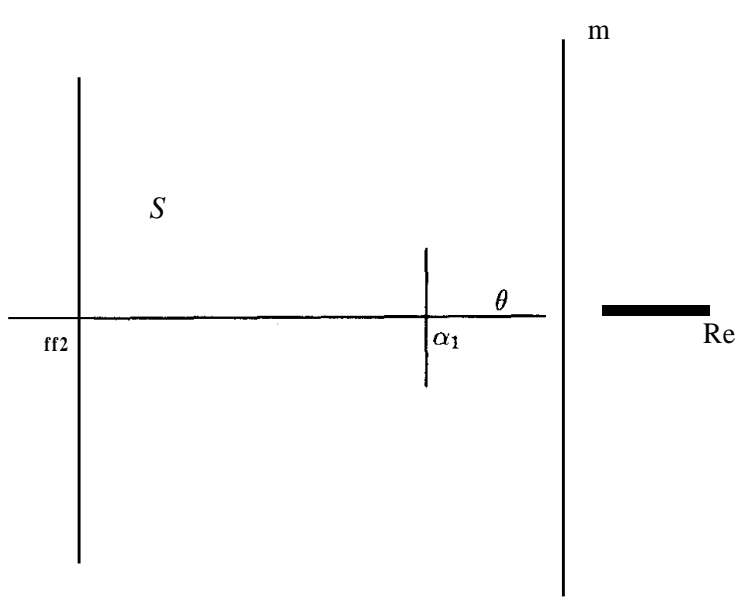

Figure 1: A popular stability region $S$ in the complex plane

\subsection{Equalities}

The feedback gain matrix It' may be constrained by the way the feedback (2.2) is realised. Static output feedback, decentralized state feedback, simultaneously stabilizing state feedback for several operating points of a system, and two-controller feedback are some examples of control structure realizations which impose constraints on $K$. Here we show how to set up equalities which correspond to the control structure constraints for the first two examples. Equalities for the other two examples can be found in [9]. Then we formulate determinantal equalities, arising from (2.5)-(2.7), which when appended to the control structure equalities, give (2.4). Let $v$ be as in Section 2.1 and $K(v)$ denote the unique value of the gain, $K$, corresponding to $(v, A, B)$ The function $K(v)$ is given by Ackermann's formula (ser [1]) which is given in the Appendix.

We first consider control structure equalities.

Static output feedback Here the feedback is realized as $u(t)=K_{o} y(t)$. We consider this problem as one of finding a linear state feedback gain vector $K$ such that the closed-loop system is S-stable with the added requirement

$$
K(v) C_{k}=0
$$

where $C_{k}=\operatorname{Ker}(C)$, i.e., $C C_{k}=0$ and $C_{k}$ has maximal column rank, $(n-p)$. We view (2.12) as an equality constraint on $v$. Once a suitable $v$ is found the output feedback matrix $K_{0}$ is given by $K_{o}=K(v) C_{r}$, where $C_{r}$ is a right inverse of $C$.

Decentralized state feedback In the case of decentralized state feedback the gain matrix $K$ is block diagonal, $K=$ block diag $\left[K_{d}^{1}, \ldots, K_{d}^{r}\right]$, where $r$ is the number of subsystems of a large-scale system. Such a feedback is popularly used for the control of large scale systems. The control structure constraints can be expressed as

$$
E_{i} K(v) \bar{E}_{i}=0, i=1,2, \ldots, r,
$$

where the matrices $E_{i}, \bar{E}_{i}$ are constant matrices o appropriate dimensions which select $i^{\text {th }}$ diagonal block of $K$.

Now we consider determinantal equalities.

The derivation of (2.9)-(2.11) can be found in [9]. 
From (2.5)-(2.7) we get

$$
\operatorname{det}(\bar{P}(s)+\Gamma V(s))=\left[\prod_{i=1}^{l}\left(s^{2}+a_{i} s+b_{i}\right) \mid(s-4)\right.
$$

(2.14) can be shown to be equivalent to a set of $n$ equalities given below. We assume that $\left(a_{i}, b_{i}\right)$ 's are distinct. We first introduce some notation:

$$
\begin{gathered}
\left.C_{i}=\begin{array}{cc}
0 & 1 \\
-b_{i} & -a_{i}
\end{array}\right], \quad e_{1}^{T}=\left[\begin{array}{ll}
1 & 0
\end{array}\right], \\
\text { block } \operatorname{det}\left(P\left(C_{i}\right)\right)=\left.\operatorname{det}(P(s))\right|_{s=C_{i}}
\end{gathered}
$$

If $\left(a_{i}, b_{i}\right)$ 's are distinct then (2.14) is equivalent to

$$
\begin{array}{r}
e_{1}^{T}\left[\text { block } \operatorname{det}\left(\bar{P}\left(C_{i}\right)+\Gamma V\left(C_{i}\right)\right]=0,\right. \\
\operatorname{det}(\bar{P}(\lambda)+\Gamma V(\lambda))=0
\end{array}
$$

We omit proof of equivalence of (2.14) and (2.15) for the sake of brevity.

In the case of static output feedback (2.12) and (2.15) define (2.4) and in the case of decentralized state feedback (2.13) and (2.15) define (2.4). In both cases the inequalities (2.9)-(2.11) which correspond to the S-stability of $(A-B K)$ define (2.3).

\section{A Homotopy method for solving a set of equalities and inequalities}

In this section, we scilve the system of equalities and inequalities obtained in the previous section by using a modified homotopy method. For a detailed discussion of honiotopy methods see [3]. Homotopy method of solving a zero finding problem $h(v)=0$, where $h: R^{N^{\prime}} \rightarrow R^{M}$ and $M \leq N$, is as follows. A homotopy $H(v, t)$ function is set up with the following properties: (a) $H(v, 1)=h(v)$; and (b) the system of equations $H(v, 0)=0$ is trivial in the sense that it is very easy to find a $\bar{v}$ satisfying $H(\bar{v}, 0)=0$. There are several examples of homotopies, i.e., ways of choosing the $H$ function. For example, consider $H(v, t)=h(v)+(t-1) h(\bar{v})$, where $\vec{v}$ is some arbitrary element of $R^{N}$, perhaps an initial guess for a solution of $h(v)=0$. This $H$ is referred to as 'Newton Homotopy' (see [3]). Conaider the zero set of $H: Z=\{(v, t): H(v, t)=0\}$. Starting from the known value, $(\bar{v}, 0) \in Z$, a numerical procedure is used to move on $Z$ until the hyperplane $t=: 1 \mathrm{js}$ met. If $\left(v^{*}, 1\right)$ is the corresponding point on $Z$ then $v^{*}$ solves $h(v)=0$.

There are two cases to be considered. In case $1, M=N$ and $Z$ is an one-dimensional curve. In case $2, M<N$, and $Z$ is an $(N-M+1)$-dimensional surface.

The classical homotispy methods consider only Case 1 . The numerical curve follower will find a solution to (3.1) if and only if there exists a smooth continuous curve connecting the two solutions, the one at $t=0$ and the other at $t=1$. Smoothness of the curve is usually easy to establish by using Sard's theorem from differential topology. It is the connectedness of the two solutions which is difficult to prove. Conditions which guarantee a solution to the problem often play a key role in the setting up of a proper homotopy and then showing connectedness. For a class of problems degree theory is useful in showing connt ctedness ( see [3]). For the class of problems which we are considering in this paper we have a proof of smoothness (which is not included in this paper) but not of coniiectedness which appeals to be difficult. However, it is observed that our method has good empirical success in finding a solution when one exists.
For homotopy methods it has been observed that the domain of attraction is usually much bigger than the one for usual it erative methods. See [14] for an example illustrating this property of homotopy methods. This is the reason for our preference of homotopy methods over other iterative methods.

The above classical homotopy approach outliner above needs modification before it can be used to solve (2.3), and (2.4). This is because of two reasons. The first is the presence $\mathrm{f}$ inequalities $g(v) \leq 0$ and the second is that $h(v)$ in (2.4) is such that $M<N$ (This can be easily seen from section 2.2). To move on $Z$ it is sufficient to choose an appropriate curve which is restricted to $\mathrm{Z}$ and then to use a curve following method to move on $Z$. "This freedom of choice to choose a curve restricted to $Z$ is utilised by adopting an optimization framework in which $(1-t)$ is taken as the objective function to be minimized. Since $t=1$ has to be reached for the homotopy method to be successful, and $t=1$ has to be reached before crossing over to $t>1,(1-t)$ is a good choice for the objective function. Thus, our selection of the one dimensional motion on $Z$ will be based on the following optimization problem :

$$
\min f(w) \quad \text { subject to } H(w)=0, w \in W
$$

where $w=(v, t), f(w)=(1-t)$ and $W=\{v: g(v) \leq 0\} \times R$. A typical numerical method for solving such an optimization problem starts from a feasible point and then iteratively generates feasible points with successive improvement in the oh jective function. For our optimization problem, $(\bar{v}, 0)$ is clearly a feasible point for the equality constraint $H(v, t)=0$. Also, $v$ can be easily chosen such that it is a feasible point for the inequalities $g(v) \leq 0$ since the inequalities are usually simple. See, for example, (2.9)-(2.11).

It is important to note that, with $(1-t)$ as the cost function, the aim is not to solve the Optimization problem (3.1). It is formulated only to drive the motion from $t=0$ to $l=1$.

Our selection of an one-dimensional curve on $\%$ is based on the continuous realization of the gradient projectioii method arid a barrier function to solve (3.1).

\section{A continuous realization of the gradient pro- jection method and a barrier method}

To have ease in presentation we first consider (3.1) with the equality constraints $H(w)=0$ only. We take up the caise of inequality constraints later. Consider the solution of (3.1) for the case, $W=R^{N+1}$. Let: $\nabla f(w)$ denote the gradient off with respect to $w$; and $H_{w}(w)$ be the Jacobian of $H$ with respect to w; and $\Omega=\{w: H(w)=0\}$.

The gradient projection method is an extension of the method of steepest descent to problems with equality constraints. The negative gradient is projected onto the tangent ssace of $\Omega$ to define the direction of movement. Let the projection of $\nabla f(w)$ onto the tangent space of $\Omega$ at w be denoted by $\nabla f_{p}(w)$ It can be shown that (see [6]) among all directions of unit euclidean length in the tangent space of $\Omega$ at $w,-\nabla f_{p}(w)$ has the maximum rate of reduction of $f(w)$. The projected gradient $\nabla f_{p}(w)$ can be written as

$$
\nabla f_{p}(w)=\nabla f(w)+H_{w}^{T}(w) \mu
$$

where,

$$
\mu=-H_{w}^{T}(w)\left[H_{w}(w) H_{w}^{T}(w)\right]^{-1} H_{w}(w) \nabla f_{p}(w)
$$

In a continuous realization (see [2]) of the gradie nt projection method the movement is described by 
The differential (3.4) equation is numerically integrated from the initial value $\mathrm{w}=(\bar{v}, 0)$ and integration is terminate 1 when $t=1$ is reached.

There are various advantages of the continuous realization (see [2]). If a solution exists for (3.4) it is smooth and thus the eccentricity problem, usually observed with the discrete version, is less significant. Also The problem of numerical integration of (3.4) is well suited to computers.

The equation (3.4) can be numerically integrated by using one of the available state-of-the-art ODE(ordinary differential equations) solving packages (see [15]). While using (3.4) for numerical integration it may happen that the trajectory generated by an ODE solving package moves away from the surface $H(w)=0$ due to error build-up and thus leads to an inaccurate solution. This error build-up can be avoided by using a DAE(differential algebraic equations), instead of an ODE, formulation as given below.

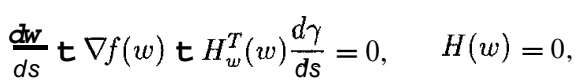

where $\frac{d \gamma}{d s}=\mu$. (3.5) explicitly takes into consideration the constraint $\stackrel{d s}{H}(w)=0$ and can be solved by using onr of the DAE solving packages (see [8]).

We now discuss a barrier method to handle tie inequality constraints. Barrier methods are used to remove the inequality constraints by modifying the objective function (nee [6]). We modify (3.1) as

$$
\min f(w)+c b(w) \quad \text { subject to } H(w)=(\mathrm{I},
$$

where

$$
b(w)=-\sum_{i=1}^{q} \ln \left(-g_{i}(v)\right)
$$

Recall that $\mathrm{w}=(v, t)$, and, $g_{i}(v) \leq 0, \mathrm{i}=1, \ldots, \mathbf{q}$ are the inequalities which define the constraint set $W$. The function $b(w)$ is called a barrier function as it establishes a barrier on the boundary of $W$. To obtain a solution of (3.1) it sequence of equality constrained problems (3.6) with $\mathrm{c} \rightarrow 0$ are solved. Recall that our aim is only to reach $t=1$ and not obtain a solution of (3.1).

An algorithm for the solution of (2.3)-(2.4), which uses homotopy, gradient projection, and barrier methods can be developed in a straightforward manner. We omit its description for the sake of brevity. We also omit the expressions for the Jscobians of $h$ and $\mathrm{g}$ with respect to $\mathrm{v}$, needed for implementing the numerical solution of (3.1). The formulae in the appendix aid their derivation.

\section{Examples}

We illustrate our method with two examples.

Example 1. Static output feedback

We consider the example of a four-state, two-input, twooutput simplified lateral directional model of the NASA F-8 DFBW airplane. The numerical data for $A, B$ and $C$ are taken from [5]. The aim is to find an output feedback gain matrix $K_{0}$ such that the closed-loop eigenvalues lie in the stability regir $\mathrm{n} S$ of fig. 1 with $\theta=45^{\circ}, \alpha_{1}=-0.2$ and $\alpha_{2}=-\infty$ OUf results on this example are tabulated in Table 1 . In comparison with the method in [5] our method requires fewer variables and does nut require any transformations on variables to handle the stabilitv region $S$ of fig. 1. For this example ODE formulation of (3.4) resulted in a sufficiently accurate solution. To numerically integrate (3.4) we
Table 1: Static output feedback

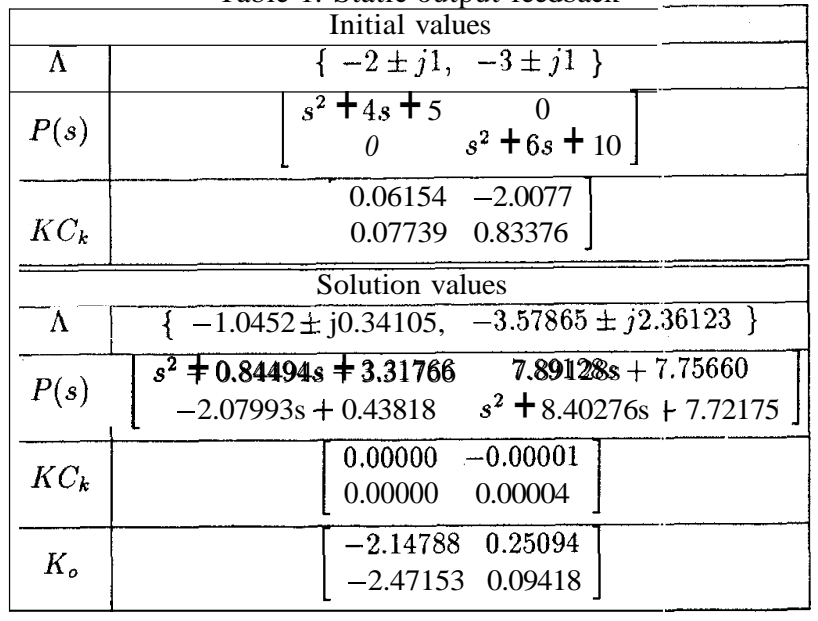

used the subroutine RDEAM from the package of ODE solvers DEPAC [15]. RDEAM solves initial value problems in ordinary differential equations using an Adams-Moulton method.

Example 2. Decentralized state feedback

We consider the example of seven-state, twa-input model of a two-area power system. The numerical data for $A, B$ matrices are taken from [7]. This seven-state model is augmented by addition of two state variables which represent integrals of the area control errors. With this augmentation the system order becomes nine. Various methods based on Lypunov fi nctions have been proposed to find decentralized state feedback law for such large-scale systems (see $[7,11,13])$. These methods guarantee stability but the closed-loop eigenvalues are not guarariteed to be in $S$. Our method takes care of the requirement of the S-stability in a very simple way. Also our method does not need any assumptions on the interconnection structure of a large scale system. The stability region $S$ is specified by $\alpha_{1}=-1, \alpha_{2}=-10$, and $\theta=45^{\circ}$. Results obtained by us on this example are tabulated in Table 2. For this example use of RDEAM to in tegrate (3.4) resulted in siginficant deviations from the constraints $H(w)=0$. So, we used the DAE formulation of (3.5) which resılted in a sufficiently accurate solution. We solved (3.5) using the subroutine DDASSL which solves a DAE by the backward differentiation formulae (see [8]).

\section{Conclusions}

In this paper, we have presented a unified numerical method to solve a general class of problems of stabilization of multivariable systems with constraints on the state feedback and the stability region. In particular, we have considered the problems of stabilization with static output feedback, and decentralized state feedback. We also have successfully worked ont examples on Sstabilization with constraints on the state feedback arising from such control structures as constant gain state feedback for several operating points of a system, and two-controller state feedback. The results on these examples are not given here due to the limitation on the number of pages. Our method first reduces a given stabilization problem into one of solving a system of equalities and inequalities. This system is then solved using the methods of gradient projection and barrier functions under $t$ he homotopy framework. Detailed tests on several problems hav s shown that the method has high degree of success. 
Table 2: Decentralized state feedback

\begin{tabular}{|c|c|}
\hline \multicolumn{2}{|r|}{ Initial values } \\
\hline$\Lambda$ & $\{-2 \pm j 1,-3 \pm j 1,-4 \pm j 1,-5 f j,-2\}$ \\
\hline$P(s)$ & $\begin{array}{l}p_{12}(s)=0 \\
p_{21}(s)=0\end{array}$ \\
\hline$E_{1} K \bar{E}_{1}$ & \\
\hline & \\
\hline A & $\begin{array}{ll}\{-1.4708 \pm j 0.6910, & -2.7249 \pm j 09920 \\
-4.4294 \pm j 1.4941, & -6.2748 \pm j 30565 \\
-1.9967) & \end{array}$ \\
\hline$P(s)$ & $\begin{aligned} p_{11}(s)= & s^{5} \mathbf{t} 13.923 s^{4} \mathbf{t} 82.351 s^{3}+271 \\
& +316 s^{2} \\
p_{12}(s)= & -3.950 s^{3}-40.961 s^{2}-101.740 .:-108.531 \\
p_{21}(s)= & -3.270 s^{3}-58.282 s^{2}-10.790 s-146.782 \\
p_{22}(s)= & s^{4} \mathbf{t} 17.873 s^{3} \mathbf{t} 116.771 s^{2} \mathbf{t} 269.408 s \\
& \quad+269.346\end{aligned}$ \\
\hline $\bar{E}_{1} K \bar{E}_{1}$ & $\begin{array}{llll}0.000 & 0.000 & 0.000 & 0.000\end{array}$ \\
\hline$E_{2} K \bar{E}_{2}$ & {$\left[\begin{array}{llll}0.000 & 0.000 & 0.000 & 0.000\end{array}\right]$} \\
\hline$K_{d}^{1}$ & $\begin{array}{lllll}0.2388 & 0.9595 & 1.8432 & 0.6432 & -0.5801\end{array}$ \\
\hline$K_{d}^{2}$ & {$\left[\begin{array}{lllll}2.7012 & 0.6376 & 1.6218 & 2.1592 & 1.0773\end{array}\right]$} \\
\hline
\end{tabular}

Acknowledgement

Prof. M. R. Chidambara's help in the prepe ration of this paper is gratefully acknowledged.

\section{References}

[1] J. Ackermann, Sampled Data Control Systems : Analysis and Synthesis, Robust System Design. Springer Veilag, Berlin, 1985.

[2] M. T. Chu, "On the continuous realization of iterative processes," SIAM Review, vol. 30, pp. 375-387, 1988.

[3] C. B Garcia, and V.I. Zangwill, Pathways to Solutions, Fixed Points and Equilibria. Prentice-Hall, Englewood Cliffs, New Jersey, 1981.

[4] T. L. Harris, R. A. DeCarlo, and S. Richter, "A continuation approach to aigenvalue assignment," Automatica, vol. 21, pp. 551-555, 1983.

[5] L H. Keel, and S.P. Bhattacharyya, "State-sp ice design of low-order stabilizers," IEEE Trans. Auto. Control, vol. 35, pp. 182-186, 1990.

[6] D. G. Lueriberger. Linear and Nonlinear Progrumming. Addison Wesley, Readirg, Mass., 1984.

[7] A. K. Mahalanabis, and R. Singh, "On decen ralized feedback stabilization of large-scale interconnected systems," Int I_Control, vol. 32, pp. 115-126, 1980.

[8] L. R. Petzold, A Description of DASSL : A differential/ algebraic system solver. Ten. Rep., SAND-82-8637, Sandia Laboratories, New Nexico, 1982.
[9] M. S. Phatak, and S. S. Keerthi, "A homotopy method for the stabilization of single input linear systems with structural constraints on the state feedback," submitted in revised form to Automatica, 1991.

[10] S. Richter, and R.A. DeCarlo, "A homotopy method for eigenvalue assignment using decentralized statie feedback.," IEEE Trans. Auto. Control, vol. AC-29, 148-158, 1984.

[11] D. D. Siljak, and M. B. Vukcevic, "Decentrally stabilizable linear and bilinear large scale systems," Int. J. Control, vol. 26, pp. 289-305, 1977.

[12] H. R. Sirisena, and S. S. Choi, "Pole placennent in prescribed regions of the complex plane using output feedback," IEEE Trans. Auto. Control, vol. AC-20, pp. 810-812, 1975.

[13] N. Viswanadharn, and A. Rarnakrishna, "Decentralized estimation and control for interconnect ed systems," Large Scale Systems, vol. 3, pp. 255-266, 1982

[14] T. L. Wayburn, and J. D. Seader, "Homotopy continuation methods for computer-aided process design," Comput. Chem. Engng., vol. 11, pp. 7-25, 198".

[15] H. A. Watts, RDEAM - An Adams ODE code with root solving capability. Ten. Rep., SAND 85-1595, Sandia Laboratories, New Mexico, 1985.

\section{Appendix : Ackermann's formula}

The unique value of $K$ for which (2.5)-(2.6) hold is given by (see [1])

$$
K=U^{-1}(\bar{K}+\Gamma T),
$$

where $\Gamma$ is $m \times n, U$ is $m \times m, \bar{K}$ is $i n \times n$, and $T$ is $n \times n$. $U$, $\mathrm{K}$, and $T$ are as given below.

Let the input matrix $B=\left[b_{1}, b_{2}, \ldots, b_{m}\right]$, and $n_{1}, n_{2}, \ldots, n_{m}$ be the controllability indices of the pair $(\mathrm{A}, B)$. Let $e_{i}$ be an $n \times 1$ column-vector with all elements zero except the $i^{\text {th }}$ one which is equal to one, and $\eta_{i}=n_{1}+n_{2}+\ldots+n_{i}$. Define

$Q_{i}=\left[b_{i}, A b_{i}, \ldots, A^{n_{i}-1} b_{i}\right], \quad \mathrm{Q}=\left[Q_{1}, Q_{2}, \ldots, Q_{m}\right], \quad \beta_{i}=e_{\eta_{i}}^{T} Q^{-1}$

Then

$$
T_{i}=\left[\begin{array}{c}
\beta_{i} \\
\beta_{i} A \\
\vdots \\
\beta_{i} A^{n_{i}-1}
\end{array}\right], T=\left[\begin{array}{c}
T_{1} \\
T_{2} \\
\vdots \\
T_{m}
\end{array}\right] ; \quad \bar{K}=\left[\begin{array}{c}
\beta_{1} A^{n_{1}} \\
\beta_{2} A^{n_{2}} \\
\vdots \\
\beta_{m} A^{n_{m}}
\end{array}\right]
$$

The matrix $T$ is a similarity transformation matrix which transforms $(\mathrm{A}, B)$ into controllable cannocical form $\left(A_{c}, b_{c}\right)$, where $\mathrm{A},=T A T-{ }^{\prime}$, and $B,=T B$. The matrix $U$ is upper triangular and is such that $\left[e_{\eta_{1}}, \mathrm{e}, \ldots, \ldots, e_{\eta_{m}}\right] U=B_{c}$.

The $K$ just defined produces the closed-loop sca'ar characteristic polynomial $\operatorname{det}(s I-A+B K)=\operatorname{det}(P(s))$, where the $\mathrm{m} \times m$ ploynomial matrix $P(s)$ is as given below. Define :

$$
\begin{gathered}
\bar{P}(s)=\operatorname{diag}\left[s^{n_{1}}, s^{n_{2}}, \ldots, s^{n_{m}}\right] \\
v_{i}(s)=\left[1, s, \ldots, s^{n_{i}-1}\right]^{T}, \quad V(s)=\left[v_{1}(s), v_{2}(s) ., v_{m_{m}}(s)\right]
\end{gathered}
$$

Then,

$$
P(s)=\bar{P}(s) \mathbf{t} \Gamma V(s) .
$$

